\title{
Effectiveness of Motivational Interviewing in Influencing Smoking Cessation in Pregnant and Postpartum Disadvantaged Women
}

\author{
Catherine B. Hayes MD, FFPHMI, MPH, MRCGP ${ }^{1,2}$, Claire Collins PhD, MSocSc, BSocSc, DipStats ${ }^{3,4}$, \\ Helen O'Carroll RGN, RM ${ }^{4}$, Emma Wyse ${ }^{4}$, Miriam Gunning RGN, RSCN ${ }^{1}$, Michael Geary MD, FRCOG, \\ FRCPI, DCH ${ }^{4}$, Cecily C. Kelleher MD, FRCPI, MPH, FFPH, FFPHMI ${ }^{5}$ \\ ${ }^{1}$ Dr Steevens' Hospital, Dublin, Ireland; ${ }^{2}$ Department of Public Health and Primary Care, Trinity College Dublin, Dublin, \\ Ireland; ${ }^{3}$ Irish College of General Practitioners, Lincoln Place, Dublin, Ireland; ${ }^{4}$ Rotunda Hospital, Dublin, Ireland; \\ ${ }_{5}^{5}$ School of Public Health, Physiotherapy, and Population Science, University College Dublin, Belfield, Dublin, Ireland \\ Corresponding Author: Catherine B. Hayes, MD, FFPHMI, MPH, Department of Public Health and Primary Care, Trinity \\ College Dublin, Trinity Center for Health Sciences, AMNCH, TALLAGHT, Dublin, Ireland. Telephone: 353(0)1 896 1087/2293; \\ Fax: 353(0)1896 4031211; E-mail: hayesc9@tcd.ie
}

Received December 22, 2011; accepted September 10, 2012

\begin{abstract}
Introduction: Systematic assessments of Motivational Interviewing (MI) in smoking behavior have been rare to date. This study aimed to determine whether an integrated approach, involving staff training in MI techniques, was sufficient to affect change in smoking status or intensity in low-income pregnant and postpartum women.

Methods: Overall, 500 consecutive smokers were recruited at first prenatal visit to public antenatal clinics. Following staff training, 500 more were recruited (intervention group). Data were recorded at 28-32 weeks gestation, after birth, at 3-4 and 7-9 months postpartum. The primary outcome measure was self-reported continued abstinence from smoking verified by urinary cotinine analysis. Changes in smoking intensity were also measured.

Results: There was no significant difference in the proportion of smokers in the intervention and control groups who reported stopping smoking at $28-32$ weeks gestation $(8.2 \%$ vs. $8.8 \% ; p=.73), 1$ week after birth $(8.6 \%$ vs. $11.4 \% ; p=.14), 3-4$ months after birth ( $5.8 \%$ vs. $4.8 \% ; p=.48)$, or $7-9$ months after birth $(5.2 \%$ vs. $4.0 \% ; p=.36)$. Although more cases were nonsmoking at the second visit, $14.8 \%[95 \% \mathrm{CI}=11.8-18.5]$ vs. $13.1 \%$ controls $[95 \% \mathrm{CI}=10.3-16.6]$, this was not statistically significant.

Conclusions: MI delivered at a number of time points during pregnancy and up to 9 months postpartum failed to affect quit rates. It may have had a small effect in preventing relapse among spontaneous quitters in late pregnancy though the validity of this remains uncertain.
\end{abstract}

\section{INTRODUCTION}

Smoking is still the commonest public health problem in pregnancy. It is one of the few potentially preventable factors associated with adverse outcomes in pregnancy, perinatally, in infancy, and in childhood (Cnattingius, 2004; Salihu $\&$ Wilson, 2007). Prevalence of smoking in Ireland is highest in young girls of childbearing age, with a distinct social class gradient (Morgan et al., 2008), and has increased over time (Friel, Nic Gabhainn, \& Kelleher, 1999; Kelleher et al., 2003; Morgan et al., 2008). This cohort of women attends public prenatal clinics during pregnancy and is a key target for intervention.

A systematic review of interventions for smoking cessation in pregnancy found that smoking cessation programs in pregnancy appeared effective in reducing smoking rates, low birth weight, and preterm birth (Lumley, Oliver, Chamberlain,
\& Oakley, 2004). An updated review, which included eight new studies (Lumley et al., 2009), similarly showed that cessation interventions in early pregnancy reduced smoking in later pregnancy by between $6 \%$ and $9 \%$. A subgroup analysis, which included cognitive behavior therapy, educational and motivational interviewing (MI) strategies, showed a pooled effect similar to that of the whole group (RR $0.95,95 \% \mathrm{CI}$ 0.93-0.97).

MI is a client-centered, directive method for enhancing intrinsic motivation to change by exploring and resolving ambivalence (Miller \& Rollnick, 1991). MI recognizes change as a natural process which is especially true in pregnancy when between $20 \%$ and $40 \%$ of pregnant smokers quit, the majority in early pregnancy (Cnattingius, 2004; Fingerhut, Kleinman, \& Kendrick, 1990) although at least two-thirds relapse in the first year after birth (Fingerhut et al., 1990; Lumley et al., 2004; Tong, Jones, Dietz, D’Angelo, \&

doi:10.1093/ntr/nts225

Advance Access publication October 29, 2012

(C) The Author 2012. Published by Oxford University Press on behalf of the Society for Research on Nicotine and Tobacco.

All rights reserved. For permissions, please e-mail: journals.permissions@oup.com 


\section{Effectiveness of motivational interviewing}

Bombard, 2009). It is recognized that even brief MI counseling can accelerate or facilitate change (Miller \& Rollnick, 1991), particularly with clients who are less motivated and who are ambivalent about changing behavior (DiClemente \& Prochaska, 1998).

The potential effectiveness of MI in addressing smoking cessation in pregnancy is biologically plausible as this group of women are similar to those with other types of addiction where MI has shown definite impact (Heil, Linares Scott, \& Higgins, 2009). Despite this, evidence around the effectiveness of MI in addressing smoking cessation in pregnancy and postpartum at the time of this study was modest, with studies showing at most short-term benefits (Dolan-Mullen et al., 2000; Stotts, DiClemente, \& Dolan-Mullen, 2002).

This article set out to provide further evidence of effectiveness of an MI intervention among a large cohort of pregnant smokers and those who had quit since becoming pregnant.

\section{METHODS}

\section{Study Design}

We used a quasi-experimental historical cohort design to determine whether MI was effective in increasing quit rates at a number of longitudinal time points in pregnancy and postpartum. This design was adopted because this was in effect a group intervention and contamination could not be avoided. Although quit rates were the primary outcome measure, we acknowledged that the goal of quitting smoking during pregnancy and staying quit might be unrealistic for this group of low-income women and hence variation in smoking intensity was also examined with a harm-reduction approach in mind (Ershoff, Solomon, \& Dolan-Mullen, 2000).

\section{Inclusion Criteria}

Expectant mothers were recruited to public prenatal clinics at first prenatal visit who were smokers at the time of pregnancy and who met the criteria for inclusion in the study (age 16-40, Irish national and resident in North Dublin City and County). A smoker at the time of pregnancy was defined as

currently smoking (at least one cigarette in past 7 days); smoked at time of pregnancy but stopped since becoming aware of pregnancy (spontaneous quitters).

This latter group was purposefully included as it is well recognized that smoking behavior in pregnancy demonstrates a complex and often unpredictable pattern (Pickett, Wakschlag, Dai, \& Leventhal, 2003). Most of those who spontaneously quit early in pregnancy relapse either before or shortly after delivery (Solomon \& Quinn, 2004). This group was thus included as smokers because of the possibility of relapse, the potential to benefit from the intervention and the focus on postpartum smoking behavior. A quitter was therefore defined as a woman who stopped smoking having become aware of pregnancy or at time of first prenatal visit and stayed nonsmoking during and after pregnancy (Abrahamsson \& Ejlertsson, 2000).

\section{Sample Size}

Lumley et al. (2004) showed pooled absolute differences in smoking cessation rates of $6.6 \%-9.2 \%$ between cases and controls, with higher estimated differences in better quality studies of higher intensity using validated smoking cessation measures. Data from a previous study of public prenatal clinics in the three maternity teaching hospitals in Dublin showed that $19 \%$ of smokers quit for the duration of pregnancy (Collins, 2002). Therefore, using a $95 \% \mathrm{CI}$ and an $80 \%$ power to detect an expected difference in quit rate of $8 \%$ between cases and controls and allowing for a $15 \%$ participant drop-out rate, a total sample size of 984 was deemed necessary and 1,000 women were recruited.

\section{Enrollment}

Two part-time researchers were employed to enroll eligible women. A presenting sample of 500 expectant mothers was recruited first and received "usual care" from hospital midwives, doctors, and, subsequently, public health nurses in the community which may or not have included advice and information on smoking cessation. Recruitment of the 500 intervention women followed a 3-month washout period during which health personnel were trained in brief intervention using MI techniques. All recruits to the study were identified by means of a sticker placed on the front of the patients, chart to facilitate further follow-up. Monthly analysis of recruitment rates was provided by written report to a Steering Committee.

\section{Training}

The training of hospital and public health nursing staff was delivered by two Health Promotion Officers who attended a course designed and delivered by an independent Consultant Midwife who specialized in MI training in pregnancy and postpartum and had delivered training to nurses at the Swedish Cancer Society, as part of the Smoke Free-children Initiative (Cancerfonden, 2001). We had planned to carry out 2 full days of training. However, it became evident that this would not be possible for front-line staff in the context of maintaining service delivery. Participants were subsequently given the choice of 2 full days, which encompassed video role-play and individual feedback or condensed 1-day training. The aim of the course was to provide staff with an understanding of the principles of MI and to develop skills in its practice within a brief time period of $5-10 \mathrm{~min}$.

The aspects of the training that were covered are shown in Table 1.

\section{Data Collection}

Demographic details at the first prenatal visit included age, marital status, number of children, medical card status, gravid status, and number of weeks pregnant. The smoking history included current smoking status (see above), number of years smoking, amount smoked, partner smoking, and number of additional smokers in the home.

\section{Outcome Measures (Including Biochemical Validation)}

Russell Standard total abstinence at each time point was used to define smoking cessation (Russell, Crawford, \& Woodby, 2004). Self-reported smoking cessation was verified by near patient urinary cotinine testing at 28-32 weeks pregnancy and 
Nicotine \& Tobacco Research, Volume 15, Number 5 (May 2013)

Table 1. Description of Training Program

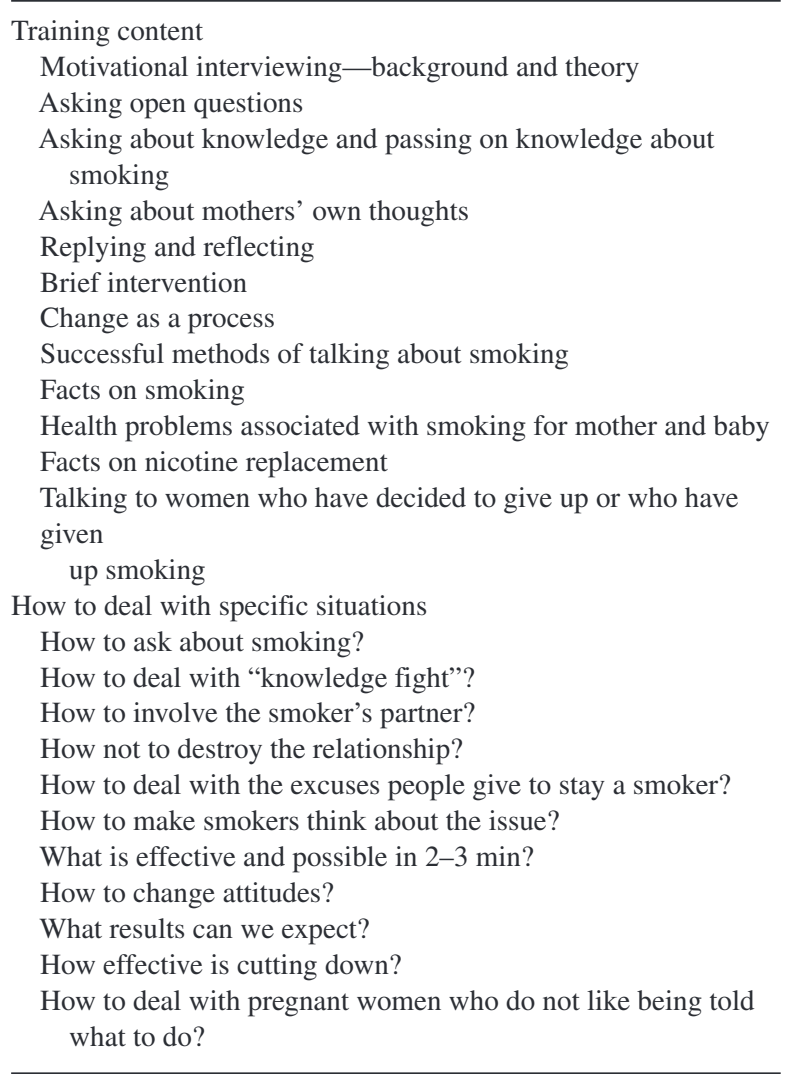

at 3-4 months postpartum. Using and interpreting the results of urinary cotinine testing using analysis by a simple colorimetric assay, the "Smokescreen" test (Cope, Nayyar, Holder, Brock, \& Chappel, 2000), formed part of the training program. With this test, when urine is mixed with reagent, the sample is positive for nicotine metabolites if it develops a redorange color. The degree of nicotine consumption (from light to very heavy) can be easily assessed using an accompanying color chart with the color becoming darker with increased nicotine intake. The primary outcome variables were (a) change in smoking status based on self-reported response and (b) urinary cotinine measurement for those who had selfreported quitting.

Smoking status was also categorized according to the number of cigarettes smoked into the following categories: None (0), Light (1-5), Medium (6-10), Heavy (11-15), and Very heavy (16+) (Pickett, Rathouz, Kasza, Wakschlag, \& Wright, 2005). Fluctuations in smoking intensity between the baseline and at 28-32 weeks pregnancy and between baseline and 3-4 months postpartum were investigated to give a picture of the variation in smoking intensity.

\section{Human Subjects Approval}

The study was approved by the Rotunda Hospital Research Ethics Committee in January 2004 (Reference number 04/01/07). Informed written consent was obtained from all participants who received an information leaflet explaining the study.

\section{Implementation of the Intervention}

Brief intervention using the MI training techniques was provided by hospital and community personnel to 500 women (the intervention group) at the antenatal first prenatal visit (12-20 weeks), 28-32 weeks gestation in the hospital, at 1 week after birth, 3-4 months and 7-9 months postdelivery in the community. Interviewer-administered questionnaires were used to record data on all participants at each visit.

Commencement of the study was delayed until 3 months after the introduction of the Workplace Smoking Legislation on March 29, 2004 (Allwright et al., 2005). A pilot study commenced in April 2004. The process of intervention delivery and the time taken to deliver the intervention (range 3-10 min) were observed carefully by the researcher midwife and adjustments made to the delivery flow chart protocol. Minor revision of the data instruments was subsequently undertaken.

An MI sheet placed inside the patient's hospital chart provided a record of the number of times the intervention was delivered in the hospital setting (minimum twice) and the signature of professional staff that delivered it. Though not quantitatively analyzed, these sheets were examined by the researchers at the end of each clinic to ensure that delivery of the intervention by the health professionals had taken place and to remove the record from the chart if a woman subsequently decided not to participate. Up to two telephone calls were made to women who did not attend for prenatal follow-up by the researchers and by the public health nurses postnatally to act as a reminder and to determine the reason if a subsequent decision not to participate was taken.

The starting date for the study was June 28, 2004. Recruitment of the control group continued until February 2005. Training of hospital and community staff took place between March and June 2006. An immediate posttraining evaluation was carried out. Follow-up assessment of the knowledge, attitude, and reported behavior of participants at 6 months posttraining was also conducted.

Recruitment to the intervention group commenced in July 2006 and continued until June 2007.

\section{Data Analysis}

SPSS version 15 was used for statistical analysis. Descriptive summaries, including means and proportions and 95\% confidence intervals (CIs), were used to describe the characteristics of the study sample, changes in the outcome variables, and number of cigarettes per day. The chi ${ }^{2}$ test was used for comparison of qualitative variables; the $t$ test for two independent samples for mean differences in quantitative normally distributed variables and the Mann-Whitney $U$ test as the nonparametric equivalent.

The preliminary analysis was carried out including and excluding those who had stopped smoking by the first prenatal visit. Mean (median) differences in number of cigarettes per day were calculated within groups based on smoking intensity to examine variability in cigarettes per day within these groups.

\section{RESULTS}

In total, 211 health professionals took the training course: $83.3 \%$ the condensed and $16.7 \%$ the two full days of 


\section{Effectiveness of motivational interviewing}

Table 2. Demographic and Smoking Variables at Baseline

\begin{tabular}{|c|c|c|c|c|c|}
\hline \multirow[b]{2}{*}{ Demographics } & \multicolumn{2}{|c|}{ Intervention } & \multicolumn{2}{|c|}{ Control } & \multirow[b]{2}{*}{$p$ value } \\
\hline & $N$ & & $N$ & & \\
\hline Median age (years) & 500 & 26.2 & 500 & 26.3 & 0.95 \\
\hline First pregnancy $(\%)$ & $171 / 500$ & 34.2 & $193 / 500$ & 38.6 & 0.15 \\
\hline Median number of weeks pregnant & 500 & 13.9 & 500 & 13.4 & $0.003 *$ \\
\hline General Medical Services card (\%) & $264 / 496$ & 52.8 & $267 / 492$ & 53.4 & 0.74 \\
\hline Married $(\%)^{\mathrm{a}}$ & $86 / 500$ & 17.2 & $81 / 500$ & 16.2 & 0.40 \\
\hline Median number of children living in same house & 500 & 1.2 & 500 & 1.2 & 0.51 \\
\hline \multicolumn{6}{|l|}{ Smoking } \\
\hline Median number of years smoking ${ }^{\mathrm{b}}$ & 498 & 10.1 & 500 & 10.3 & 0.51 \\
\hline Median number of cigarettes per day & 500 & 10.2 & 500 & 9.5 & 0.16 \\
\hline Partner smoking $\%^{\mathrm{c}}$ & $327 / 470$ & 69.6 & $330 / 470$ & 70.2 & 0.83 \\
\hline Median number of cigarettes smoked/day by partner & 327 & 14.7 & 330 & 15.2 & 0.48 \\
\hline $\begin{array}{l}\text { Median number of people living in house (other than self } \\
\text { or partner) who smoke }\end{array}$ & 497 & 0.5 & 500 & 0.6 & 0.41 \\
\hline \multicolumn{6}{|l|}{ Current smoking status (\%) } \\
\hline & 500 & & 500 & & \\
\hline I smoke now & 118 & 23.6 & 209 & 18.2 & \\
\hline I smoke now but have cut down since becoming pregnant & 332 & 66.4 & 664 & 66.4 & \\
\hline I have stopped smoking since I thought I might be pregnant & 50 & 10.0 & 127 & 15.4 & $0.01 *$ \\
\hline \multicolumn{6}{|l|}{ Number of cigarettes per day $(\%)$} \\
\hline & 500 & & 500 & & \\
\hline None & 50 & 10.0 & 74 & 14.8 & $0.02 *$ \\
\hline Light (1-5) & 97 & 19.4 & 100 & 20.0 & 0.81 \\
\hline Medium (6-10) & 187 & 37.4 & 170 & 34.0 & 0.26 \\
\hline Heavy (11-15) & 72 & 14.4 & 82 & 16.4 & 0.38 \\
\hline Very heavy (16+) & 94 & 18.8 & 74 & 14.8 & 0.09 \\
\hline
\end{tabular}

aLegal marital status.

${ }^{b}$ For those still smoking at first prenatal visit.

${ }^{\mathrm{c}}$ For those who reported having a partner.

$* p<.05$.

training. Participation was high with a completion rate of $92.8 \%$. Immediate posttraining evaluation showed that skills practice was rated as excellent or good by $92 \%$. Follow-up assessment of the knowledge, attitude, and reported behavior of participants at 6 months posttraining showed that $94 \%$ felt "moderately" or "completely" able to integrate MI skills into their practice (Hayes, 2009). It also showed that participants were more confident in their ability to deliver basic MI skills and more likely to be proficient in specific MI skills compared with a control group who did not receive training.

There were $34(6.8 \%)$ initial refusals among controls and $49(9.8 \%)$ in the intervention study participants. In total, 71 women, 48 intervention (10.6\%) and 23 controls (4.9\%), refused to take part at subsequent visits. None refused to provide urinary samples for cotinine analysis. Review of the MI sheets in the charts showed a high completion rate except where women had failed to return or refused to participate at a follow-up visit.

Table 1 shows that the demographic variables were similar in both groups apart from the intervention group being half a week later in pregnancy.

Table 2 shows the smoking status of participants and the number of cigarettes smoked at baseline. A significant difference in smoking status was noted, with more spontaneous quitters in the control and more current smokers in the intervention group $\left(\mathrm{chi}^{2}=9.3, d f=2, p=.01\right)$. When spontaneous quitters were considered separately, cases were still half a week later in pregnancy (14.0 vs. 13.3 weeks); however, the mean difference was no longer statistically significant $(p=.08)$.

Table 3 shows the proportion of women who stopped smoking at the second and subsequent visits. There was no significant difference in the proportion of the total cohort who quit at each visit. When the spontaneous quitters were excluded, the nonsignificant differences remained.

A high proportion of the cohort attended at the second visit (93\% intervention vs. 94.6\% control). Difference in response rates emerged in favor of the controls from the third visit onwards. These differences were statistically significant after birth $(63.2 \%$ intervention vs. $67.2 \%$ control, $p=.009)$ and of borderline significance at 3-4 months $(55.6 . \%$ vs. $58.8 \%$, $p=0.05)$ and at $7-9$ months postpartum (47\% vs. $53.4 \%$, $p=0.05)$.

The mean number of self-reported cigarettes smoked at the first prenatal visit (V1) and late pregnancy (V2), and at 3-4 months postpartum (V4) for cases and controls within each smoking category are shown in Table 4 . There was little variation in the number of cigarettes smoked with no statistically significant differences noted.

Although more cases were nonsmoking at V2, 14.8\% [95\% $\mathrm{CI}=11.8-18.5]$ vs. $13.1 \%$ controls $[95 \% \mathrm{CI}=10.3-16.6]$, the difference was not statistically significant. A smaller proportion of cases were heavy and very heavy smokers at V4, 
Nicotine \& Tobacco Research, Volume 15, Number 5 (May 2013)

Table 3. Proportion of Women Who Had Stopped Smoking at Each Visit

\begin{tabular}{|c|c|c|c|c|c|c|c|c|c|c|}
\hline & \multicolumn{4}{|c|}{ Total cohort } & \multicolumn{5}{|c|}{ Excluding spontaneous quitters } & \multirow[b]{3}{*}{$p$} \\
\hline & \multicolumn{2}{|c|}{ Intervention } & \multicolumn{2}{|c|}{ Control } & \multirow[b]{2}{*}{$p$} & \multicolumn{2}{|c|}{ Intervention } & \multicolumn{2}{|c|}{ Control } & \\
\hline & $N=500$ & $\%$ & $N=500$ & $\%$ & & $N=450$ & $\%$ & $N=423$ & $\%$ & \\
\hline First prenatal visit & 50 & 10 & 77 & 15.4 & $0.01 *$ & & & & & \\
\hline $28-32$ weeks gestation & 41 & 8.2 & 44 & 8.8 & 0.73 & 33 & 7.3 & 22 & 5.2 & 0.20 \\
\hline 1 week after birth & 43 & 8.6 & 57 & 11.4 & 0.14 & 26 & 5.8 & 28 & 6.6 & 0.61 \\
\hline 3-4 months after birth & 29 & 5.8 & 24 & 4.8 & 0.48 & 20 & 4.4 & 10 & 2.4 & 0.09 \\
\hline 7-9 months after birth & 26 & 5.2 & 20 & 4.0 & 0.36 & 11 & 3.3 & 13 & 3.1 & 0.83 \\
\hline
\end{tabular}

$* p<0.05$

Table 4. Mean Number of Cigarettes Smoked Per Day Within Smoking Categories at V1 (Enrollment), V2 (28-32 Weeks), and V4 (3-4 Months Postpartum)

\begin{tabular}{|c|c|c|c|c|c|c|c|c|c|}
\hline & \multicolumn{3}{|r|}{ V1 } & \multicolumn{3}{|c|}{$\mathrm{V} 2$} & \multicolumn{3}{|c|}{ V4 } \\
\hline & $\mathrm{N} 1^{\mathrm{a}}$ & $\%$ & $\begin{array}{l}\text { Mean number of } \\
\text { cigarettes per day }\end{array}$ & $\mathrm{N} 2^{\mathrm{a}}$ & $\%$ & $\begin{array}{l}\text { Mean number of } \\
\text { cigarettes per day }\end{array}$ & $\mathrm{N} 4^{\mathrm{a}}$ & $\%$ & $\begin{array}{l}\text { Mean number of } \\
\text { cigarettes per day }\end{array}$ \\
\hline \multicolumn{10}{|l|}{ None } \\
\hline Case & 50 & $(10.0)$ & & 69 & (14.8) & & 20 & $(7.6)$ & \\
\hline Control & 74 & $(14.8)$ & & 62 & (13.1) & & 22 & $(7.2)$ & \\
\hline \multicolumn{10}{|l|}{ Light (1-5) } \\
\hline Case & 97 & (19.4) & 3.79 & 106 & $(22.8)$ & 3.54 & 48 & (18.4) & 4.02 \\
\hline Control & 100 & $(20.0)$ & 3.82 & 130 & $(27.5)$ & 3.28 & 62 & (20.3) & 3.71 \\
\hline \multicolumn{10}{|c|}{ Medium (6-10) } \\
\hline Case & 187 & $(37.4)$ & 8.94 & 148 & $(31.8)$ & 8.96 & 118 & $(45.0)$ & 9.03 \\
\hline Control & 170 & $(34.0)$ & 9.02 & 160 & $(33.8)$ & 8.90 & 107 & $(35.0)$ & 9.04 \\
\hline \multicolumn{10}{|c|}{ Heavy (11-15) } \\
\hline Case & 72 & (14.4) & 14.39 & 78 & (16.8) & 14.09 & 37 & (14.1) & 14.24 \\
\hline Control & 82 & (16.4) & 14.59 & 77 & (16.3) & 14.36 & 56 & (18.3) & 14.52 \\
\hline \multicolumn{10}{|c|}{ Very heavy $(16+)$} \\
\hline Case & 94 & $(18.8)$ & 21.43 & 64 & (13.8) & 22.88 & 39 & (14.9) & 23.21 \\
\hline Control & 74 & (14.8) & 22.32 & 44 & $(9.3)$ & 23.52 & 59 & (19.2) & 21.32 \\
\hline \multicolumn{10}{|l|}{ Total } \\
\hline Case & 500 & (100) & & 465 & (100) & & 262 & (100) & \\
\hline Control & 500 & (100) & & 473 & (100) & & 306 & (100) & \\
\hline
\end{tabular}

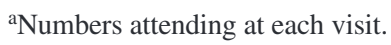

$29 \%[95 \% \mathrm{CI}=23.7-35.0]$ vs. $37.5 \%[95 \% \mathrm{CI}=32.2-43.3]$; however, the overall numbers were small and statistical significance not attained (Table 4).

Table 4 also shows a picture of postpregnancy relapse at 3-4 months postpartum for those who were nonsmoking at first prenatal visit. No differences between cases and controls were observed.

\section{CONCLUSIONS}

\section{Main Findings}

This study found that methodical delivery of good quality MI by front-line hospital and public health nursing staff to a large cohort of low-income women in pregnancy and postpartum did not affect quit rates, either for the total cohort who were smokers at time of pregnancy or for the subgroup of women who had reported stopping smoking at time of the first prenatal visit.
Although other studies had shown some impact in late pregnancy using a variety of methods (Stotts et al., 2002; Tappin et al., 2000), sustained differences between intervention and control groups have not been found (Stotts, DeLaune, Schmitz, \& Grabowski, 2004; Stotts et al., 2002; Suplee, 2005; Thyrian et al., 2006). Although fewer cases who were spontaneous quitters relapsed in late pregnancy, statistical significance was not attained hence the validity of this is uncertain. Over half of women attending the public prenatal clinics in our study had means-tested General Medical Services cards in contrast to $22 \%$ of all comparably aged women (Health Service Executive, 2008). A recent Glasgow-based RCT of home-based MI by midwives proved ineffective in improving smoking cessation rates among deprived pregnant women (Tappin et al., 2005). Our study is similar in that 2-5 sessions of MI were offered, though our staff had limited time, sometimes less than $5 \mathrm{~min}$, to interact and deliver the intervention compared with 30-min sessions offered in Glasgow. Lumley et al. (2009) reported 


\section{Effectiveness of motivational interviewing}

higher cessation rates from more intensive trials. However, the greater intensity of MI sessions in Glasgow was still ineffectual (Tappin et al., 2005).

MI in combination with other strategies has since been shown to be beneficial. A pilot study offering MI and nicotine replacement therapy (NRT) by a midwife to rural disadvantaged women in their homes, has shown that $12.7 \%$ were nonsmokers at 12 months (Bryce, Butler, Gnich, Sheehy, \& Tappin, 2007). Although use of NRT was considered for our study, there was insufficient evidence at the time to support its use.

The New England Smoking Cessation/Reduction in Pregnancy Trial (NE SCRIPT) showed that telephone-assisted MI in addition to two other interventions (self-help manuals and "Quit and Win" monetary incentive lottery) resulted in a higher quit rate for women receiving calls (Parker et al., 2007).

The US Clinical Practice Guidelines (Fiore, Bailey, \& Cohen, 2000; Fiore et al., 2008) recommend some type of behavioral counseling and medication for a variety of populations including pregnant women, for those willing to quit except where medication use is contraindicated. For those unwilling to quit, brief intervention consistent with MI strategies designed to improve motivation is recommended.

A meta-analysis of five studies of pregnant and postpartum women (Heckman, Egleston, \& Hofmann, 2010) found no difference in women who received MI compared with controls, although increased quit rates were found for the general population. Another meta-analysis of eight studies of MI in pregnancy (Hettema \& Hendricks, 2010) also showed nonsignificant abstinence rates for pregnant and postpartum women and suggest that MI may be most useful for those with low motivation and low levels of tobacco dependence, previously noted also by Prochaska, Velicer, DiClemente, and Fava (1988).

Bauld and Coleman (2009) in their briefing paper for National Institute for Clinical Excellence (NICE) concluded that effective smoking cessation interventions in pregnancy are those which use cognitive behavioral approaches including MI (Lumley et al., 2009), self-help (Naughton, Prevost, \& Sutton, 2008), and financial incentives (Bauld \& Coleman, 2009; Lumley et al., 2009). A recent qualitative U.K.-based study determined women's experiences of trying to stop smoking during pregnancy, within and outside of a financial incentives scheme (Mantzari, Florian, \& Marteau, 2012) and cautioned against directly attributing the effects of such schemes to incentives per se, as other complex intermediary pathways may be at play such as better engagement and provision of support services.

Results from the Smoking, Nicotine and Pregnancy (SNAP) trial showed that addition of a nicotine-replacement patch to behavioral support failed to show an increase in abstinence rates among pregnant women (unadjusted odds ratio 1.26, 95\% CI 0.82-1.96) (Coleman et al., 2012). Compliance was low however, with only $7.2 \%$ using the patch for longer than a month.

\section{What This Study Adds}

This study has a number of strengths including an intervention training program that was well defined, a high fidelity of training implementation, adequate sample size, and biochemical confirmation of cessation. There was a high acceptance of the intervention by participants and high response rates though differences in favor of the control group emerged after birth.
The UK national guidelines (NICE, 2006), recommended that research be undertaken to determine which brief interventions are most effective for low-income pregnant women; this study makes a positive contribution to this question.

The longitudinal study also afforded the opportunity to expand on Pickett's work which has shown that smoking behavior demonstrates substantial within person variation over the course of pregnancy (Pickett et al., 2003, 2005). Understanding this complex pattern is important, both in relation to effects on the fetus of differential exposure to cigarette smoke across three trimesters and for targeting smoking cessation interventions. A prospective study of 304 pregnant women, where smoking was measured by self-report and biochemical measures, showed that second- and third-trimester smoking predicted decrease in birth-weight with third-trimester smoking showing the greatest effect which was dose related (Espy et al., 2010).

Validation of self-reports of nonsmoking status in pregnancy, using biochemical markers, has shown repeatedly that pregnant women tend to conceal their smoking, particularly in relation to the number of cigarettes smoked (Klebanoff, Levine, Clemens, DerSimonian, \& Wilkins, 1998; Webb, Boyd, Messina, \& Windsor, 2003). Prevalence rates of at least $20 \%$ have been consistently reported by those who state at first prenatal visit that they have quit since becoming pregnant (Windsor, 2011). Including these "spontaneous quitters" in our study was thus justified, as also evidenced by the high relapse rates at 3-4 months after birth for this group as previously noted.

An accompanying qualitative study of 22 women who received the intervention found that the program had a reenforcing and facilitatory role (Hayes, 2009).

\section{Study Limitations}

The study took place prior to publication of the Motivational Interviewing Treatment Integrity (MITI) code for assessing interviewer behavior (Moyers, Martin, Manuel, Hendrickson, $\&$ Miller, 2005). The difficulties encountered in assessing the fidelity of implementation to the principles of MI for any given intervention, including the paucity of reliable and practical instruments with which to assess training, quality, and fidelity, have been noted previously (Allsop, 2007). Although a record of delivery of the intervention was meticulously maintained and checked by the researchers, and posttraining evaluation immediately and at 6 months later indicated a high degree of competence, the intervention delivery was not directly observed.

The study design was a time-based before-after study using a quasi-experimental approach. Locating the study in parallel settings at the outset would have facilitated simultaneous randomization and a halving of the recruitment period; however, informal investigation suggested that for practical reasons this would not be feasible. Training a portion of the staff in the single hospital site could potentially result in cross-contamination. Therefore, in a single setting, with the midwife as the unit of intervention, where blindness was not possible to achieve and where cross-contamination would be unavoidable and not ethical in the context of patient care, the quasi-experimental historical cohort design was deemed the most appropriate methodology.

Although the design was relatively successfully implemented, some differences arose because of the noncontemporaneous nature of the recruitment. 
There were important differences in smoking habits between the two groups at baseline with more spontaneous quitters in the control group. A possible explanation is that legislation protecting workers from the health ill effects of exposure to second-hand smoke was introduced (Allwright et al., 2005) just 3 months prior to commencement of our study; therefore, its impact had time to become established when the intervention group was recruited over a year later. This may have resulted in more committed smokers being recruited into the intervention group. The intervention group also took longer to recruit than the control group, which may suggest that the intervention group was a difficult one in which to succeed in terms of smoking cessation.

The risk of reporting no difference when there can be one (type II error) must be considered. The percentage of those who would likely have quit due to the intervention was estimated at $8 \%$, midway between the estimates reported by Lumley et al. (2004). Tappin et al. (2005) considered that the change in quit rate of 6 in 100 women continuing to smoke between intervention and control group as suggested by Lumley et al. (2004) was probably not feasible in Glasgow, an area with significant material deprivation. As our population is similar, the effect estimate chosen, although valid, may also have been too high for this group of materially disadvantaged women. However, it seems unlikely that a "true" intervention effect had been missed in view of the lack of statistical significance at each time point and if so, the size of the effect would be very small.

\section{Future Direction}

According to NICE (2008), pregnant women must be encouraged to quit smoking. The nicotine-assisted reduction to stop strategy is not considered an appropriate smoking cessation method. For low-income women, a multifaceted strategy is necessary including behavioral and improved social supports and a range of fiscal and environmental measures.

In conclusion, the brief intervention of the type and intensity offered in this study was ineffective in affecting quit rates in pregnancy and postpartum. Although there were some limitations around the study design, it is unlikely that, even in the absence of these limitations, the intervention on its own would have had measurable impact on quit rates.

\section{FUNDING}

This work was supported by a grant from the Irish Heart Foundation and the Health Service Executive and the Health Promoting Hospitals Programme.

\section{DECLARATION OF INTERESTS}

None declared.

\section{ACKNOWLEDGMENTS}

Sincere thanks is due to the women who participated in the study; the hospital and public health nursing staff who delivered the intervention; the health promotion staff who delivered the training; the Directors of Nursing and Assistant Directors of Nursing in the Health Service Executive; and researchers, management, and admin staff at the Rotunda Hospital and Health Service Executive.

\section{REFERENCES}

Abrahamsson, A., \& Ejlertsson, G. (2000). Smoking patterns during pregnancy: Differences in socioeconomic and healthrelated variables. European Journal of Public Health, 10(3), 208-213. doi:210.1093/eurpub/1010.1093.1208

Allsop, S. (2007). What is this thing called motivational interviewing? Addiction, 102, 343-345.

Allwright, S., Paul, G., Greiner, B., Mullally, B. J., Pursell, L., Kelly, A., . . P Perry, I. J. (2005). Legislation for smoke-free workplaces and health of bar workers in Ireland: Before and after study. British Medical Journal, 331(7525), 1117-1123. doi:10.1136/bmj.38636.499225.55

Bauld, L., \& Coleman, T. (2009). The effectiveness of smoking cessation interventions during pregnancy: A briefing paper. Bath and Nottingham: University of Bath.

Bryce, A., Butler, C., Gnich, W., Sheehy, C., \& Tappin, D. (2007). CATCH: Development of a home-based midwifery intervention to support young pregnant smokers to quit. Midwifery, 25, 473-482. doi:410.1016/j.midw.2007.1010.1006

Cancerfonden. (2001). Smoke free children - A report. The first 10 years. Stockholm, Sweden: National Institute of Public Health.

Cnattingius, S. (2004). The epidemiology of smoking during pregnancy: Smoking prevalence, maternal characteristics, and pregnancy outcomes. Nicotine \& Tobacco Research, 6, S125-S140. doi:10.1080/14622200410001669187

Coleman, T., Cooper, S., Thornton, J. G., Grainge, M. J., Watts, K., Britton, J., \& Lewis, S. (2012). A randomized trial of nicotine-replacement therapy patches in pregnancy. New England Journal of Medicine, 366, 808-818. doi:10.1056/ NEJMoa1109582

Collins, C. (2002). Public patient antenatal care and childbirth in Dublin: Views of service providers and users $(\mathrm{PhD})$. University College Dublin, National University of Ireland, Dublin.

Cope, G. F., Nayyar, P., Holder, R., Brock, G., \& Chappel, I. (2000). Near patient test for nicotine and its metabolites in salivatoassess smoking habit.AnnalsofClinicalBiochemistry, 37(5), 666-673. doi:610.1258/0004563001899717

DiClemente, C. C., \& Prochaska, J. O. (1998). Toward a comprehensive, transtheoretical model of change: Stages of change and addictive behaviors. In W. R. Miller \& N. E. Heather (Eds.), Treating addictive behaviors (2nd ed., pp. 3-24). New York: Plenum.

Dolan-Mullen, P., DiClemente, C. C., Velasquez, M. M., Timpson, S. C., Groff, J. Y., Carbonari, J. P., \& Nicol, L. (2000). Enhanced prenatal case management for low income smokers. Tobacco Control, 9(Suppl. 3), 75 iii-77. doi:10.1136/tc.9.suppl_3.iii75

Ershoff, D. H., Solomon, L. J., \& Dolan-Mullen, P. (2000). Predictors of intentions to stop smoking early in prenatal care. Tobacco Control, 9(Suppl. 3), iii41-iii45. doi:10.1136/ tc.1139.suppl_1133.iii1141

Espy, K. A., Fang, H., Johnson, C., Stopp, C., Wiebe, S. A., \& Respass, J. (2010). Prenatal tobacco exposure: Developmental outcomes in the neonatal period. Developmental Psychology, 47(1), 153-169. doi:110.1037/a0020724

Fingerhut, L. A., Kleinman, J. C., \& Kendrick, J. S. (1990). Smoking, before during and after pregnancy. American Journal of Public Health, 80(5), 541-544.

Fiore, M. C., Bailey, W. C., \& Cohen, S. e. a. (2000). The tobacco use and dependence clinical practice guideline panel, staff, and consortium representatives. A clinical practice guideline for treating tobacco use and dependence: A US public health service report. The Journal of the American Medical Association, 283(24), 3244-3254. 
Fiore, M. C., Jaén, C. R., Baker, T. B., Bailey, W. C., Benowitz, N. L., \& Curry, S. J. (2008). Treating tobacco use and dependence: 2008 update. Clinical practice guideline. Rockville, MD: U.S. Department of Health and Human Services.

Friel, S., Nic Gabhainn, S., \& Kelleher, C. (1999). The National Health and Lifestyle Surveys. Survey of Lifestyle, Attitudes and Nutrition, (SLÁN) \& The Irish Health Behaviour in School-Aged Children Survey (HBSC). Dublin: Department of Health and Children.

Hayes, C. (2009). How effective is Motivational Interviewing Training in influencing smoking cessation in pregnant and post-partum women in lower socio-economic groups? (Doctor of Medicine MD thesis). University College Dublin, Dublin.

Health Service Executive. (2008). Primary Care Reimbursement Service. Statistical analysis of claims and payments. Dublin: Health Service Executive.

Heckman, C. J., Egleston, B. L., \& Hofmann, M. T. (2010). Efficacy of motivational interviewing for smoking cessation: A systematic review and meta-analysis. Tobacco Control, 19(5), 410-416. doi:10.1136/tc.2009.033175

Heil, S. H., Linares Scott, T., \& Higgins, S. T. (2009). An overview of principles of effective treatment of substance use disorders and their potential application to pregnant cigarette smokers. Drug and Alcohol Dependence, 104(S1), S106-S114. doi:10.1016/j.drugalcdep.2009.05.012

Hettema, J. E., \& Hendricks, P. S. (2010). Motivational interviewing for smoking cessation: A meta-analytic review. Journal of Consulting and Clinical Psychology, 78(6), 868884. doi:10.1037/a0021498

Kelleher, C., Friel, S., Nic Gabhainn, S., Corrigan, H., Nolan, G., Sixsmith, J., . . . Cooke, M. (2003). The National Health and Lifestyle Surveys. Survey of Lifestyle, Attitudes and Nutrition, (SLÁN) \& The Irish Health Behaviour in School-Aged Children Survey (HBSC) Centre for Health Promotion Studies, Galway. Department of Health and Children.

Klebanoff, M. A., Levine, R. J., Clemens, J. D., DerSimonian, R., \& Wilkins, D. G. (1998). Serum Cotinine Concentration and Self-reported Smoking during Pregnancy. American Journal of Epidemiology, 148(3), 259-262. doi:10.1093/ oxfordjournals.aje.a009633

Lumley, J., Chamberlain, C., Dowswell, T., Oliver, S., Oakley, L., \& Watson, L. (2009). Interventions for promoting smoking cessation during pregnancy [Review]. Cochrane Database of Systematic Reviews. doi:10.1002/14651858. CD001055.pub3

Lumley, J., Oliver, S. S., Chamberlain, C., \& Oakley, L. (2004). Interventions for promoting smoking cessation during pregnancy. Cochrane Database of Systematic Reviews. doi:10.1002/14651858.CD001055.pub2

Mantzari, E., Florian, V., \& Marteau, T. (2012). The effectiveness of financial incentives for smoking cessation during pregnancy: Is it from being paid or from the extra aid?. BioMedCentral Pregnancy and Childbirth, 12, 24. doi:10.1186/1471-2393-12-24

Miller, W. R., \& Rollnick, S. (1991). Motivational interviewing: Preparing people to change addictive behaviors. New York, NY: Guildford Press.

Morgan, K., McGee, H., Watson, D., Perry, I., Barry, M., Shelley, E., . . B Brugha, R. (2008). SLÁN 2007: Survey of Lifestyle, Attitudes \& Nutrition in Ireland. Main Report. Dublin: Department of Health and Children.

Moyers, T. B., Martin, T., Manuel, J. K., Hendrickson, S. M., Miller, W. R. (2005). Assessing competence in the use of motivational interviewing. Journal of Substance Abuse Treatment, 28(1), 19-26. doi:10.1016/j.jsat.2004.11.001
Naughton, F., Prevost, A. T., \& Sutton, S. (2008). Self-help smoking cessation interventions in pregnancy: A systematic review and meta-analysis. Addiction, 103(4), 566-579. doi:10.1111/j.1360-0443.2008.02140.x

NICE. (2006). Public Health Intervention Guidance No.1. Brief interventions and referral for smoking cessation in primary care and other settings. London: National Institute for Health and Clinical Excellence.

NICE. (2008). Public Health Intervention Guidance no. 10. Smoking cessation services in primary care, pharmacies, local authorities and workplaces, particularly for manual working groups, pregnant women and hard to reach communities. London: National Institute for Health and Clinical Excellence.

Parker, D. R., Windsor, R. A., Roberts, M. B., Hecht, J., Hardy, N. V., Strolla, L. O., \& Lasater, T. M. (2007). Feasibility, cost, and cost-effectiveness of a telephone-based motivational intervention for underserved pregnant smokers. Nicotine \& Tobacco Research: Official Journal of The Society for Research on Nicotine And Tobacco, 9(10), 10431105. doi:1010.1080/14622200701591617

Pickett, K. E., Rathouz, P. J., Kasza, K., Wakschlag, L. S., \& Wright, R. (2005). Self-reported smoking, cotinine levels, and patterns of smoking in pregnancy. Paediatric and Perinatal Epidemiology, 19(5), 368-376. doi:310.1111/j.1365-3016.2005.00660.x

Pickett, K. E., Wakschlag, L. S., Dai, L., \& Leventhal, B. L. (2003). Fluctuations of Maternal Smoking During Pregnancy. Obstetrics \& Gynecology, 101(1), 140-147. doi:10.1016/S0029-7844(02)02370-0

Prochaska, J., Velicer, W. F., DiClemente, C., \& Fava, J. (1988). Measuring process of change: Applications to the cessation of smoking. American Psychological Association, 56(4), 520-528.

Russell, T., Crawford, M., \& Woodby, L. (2004). Measurements for active cigarette smoke exposure in prevalence and cessation studies: Why simply asking pregnant women isn't enough. Nicotine \& Tobacco Research, 6(Suppl. 2), S141S151. doi:110.1080/14622200410001669141

Salihu, H. M., \& Wilson, R. E. (2007). Epidemiology of prenatal smoking and perinatal outcomes. Early Human Development, 83, 713-720. doi:10.1016/j.earlhumdev.2007.08.002IISSN 0378-3782

Solomon, L., \& Quinn, V. (2004). Spontaneous quitting: Selfinitiated smoking cessation in early pregnancy. Nicotine \& Tobacco Research, 6(Suppl. 2), S203-S216. doi:10.1080/14 622200410001669132

Stotts, A. L., DeLaune, K. A., Schmitz, J. M., \& Grabowski, J. (2004). Impact of a motivational intervention on mechanisms of change in low-income pregnant smokers. Addictive Behaviors, 29(8), 1649-1657. doi:10.1016/j. addbeh.2004.02.063

Stotts, A. L., DiClemente, C. C., \& Dolan-Mullen, P. (2002). One-to-One: A motivational intervention for resistant pregnant smokers. Addictive Behaviors, 27(2), 275-292. doi:10.1016/S0306-4603(01)00174-5

Suplee, P. D. (2005). The Importance of Providing Smoking Relapse Counseling During the Postpartum Hospitalization. Journal of Obstetric, Gynecologic, \& Neonatal Nursing, 34(6), 703-712. doi:10.1177/0884217505281861

Tappin, D. M., Lumsden, M. A., Gilmour, W. H., Crawford, F., McIntyre, D., Stone, D. H., . . . Mohammed, E. (2005). Randomised controlled trial of home based motivational interviewing by midwives to help pregnant smokers quit or cut down. British Medical Journal, 331(7513), 373-377. doi:10.1136/bmj.331.7513.373

Tappin, D. M., Lumsden, M. A., McKay, C., McIntyre, D., Gilmour, H., Webber, R., . . . Currie, F. (2000). The effect 
of home-based motivational interviewing on the smoking behaviour of pregnant women: A pilot randomized controlled efficacy study. Ambulatory Child Health, 6, 34-35. doi:10.1046/j.1467-0658.2000.00015.x

Thyrian, J. R., Hannover, W., Grempler, J., Roske, K., John, U., \& Hapke, U. (2006). An Intervention to Support Postpartum Women to Quit Smoking or Remain Smoke-Free. Journal of Midwifery \& Women's Health, 51(1), 45-50.

Tong, V. T., Jones, J. R., Dietz, P. M., D’Angelo, D., \& Bombard, J. M. (2009). Trends in smoking before, during, and after pregnancy - Pregnancy Risk Assessment Monitoring System (PRAMS), United States, 31 sites, 2000--2005. Morbidity and Mortality Weekly Review, 58(SS04), 1-29.
Webb, D. A., Boyd, N. R., Messina, D., \& Windsor, R. A. (2003). The discrepancy between self-reported smoking status and urine cotinine levels among women enrolled in prenatal care at four publicly funded clinical sites. Journal of Public Health Management \& Practice, 9(4), 322.

Windsor, R. A. (2011). Behavioral treatment methods for pregnant smokers: The evidence base for prenatal care programs and professional practice. In A. Handler, J. Kennelly, \& N. E. Peacock (Eds.), Reducing racial/ethnic disparities in reproductive and perinatal outcomes. The evidence from population-based interventions (pp. 243-244). Chicago, IL: Springer. doi:10.1007/978-1-4419-1499-6 